\title{
Early-Universe and Evolution of the Present Universe: Exact Solution Models
}

\author{
Koijam Manihar Singh ${ }^{1}$, Thiyam Jairam Singh ${ }^{2}$, Kangujam Priyokumar Singh ${ }^{3}$ \\ ${ }^{1}$ Department of Mathematics, Sambalpur University, Orissa, India \\ ${ }^{2}$ Department of Mathematics, Manipur University, Imphal, India \\ ${ }^{3}$ Department of Mathematics, Ideal Girls' College, (Manipur University), Imphal, India \\ E-mail:\{drmanihar,jairamth\}@rediffmail.com,pk_mathematics@yahoo.co.in \\ Received February 17, 2011; revised April 2, 2011; accepted May 4, 2011
}

\begin{abstract}
The evolution of the Robertson-Walker type universes consisting of radiating perfect fluid distribution coupled with zero-mass scalar field in which the gravitational parameter $G$ varies with cosmic time $t$ are studied. Unified descriptions of the early evolution of the universe consisting of different phases are investigated. The different properties of the cosmological solutions are discussed and the physical behaviour of the model universes during the radiation-dominated era and also during the big bang scenario are studied. Here we obtain models which are geometrically closed and are thereby ever expanding and evolve from rest from a nonsingular hot origin with maximum (finite) energy density and temperature and a small minimum (non-zero) gravitational coupling $G$.
\end{abstract}

Keywords: Evolution, Early Universe, Inflation, Radiation-Dominated Era, Big Bang, Phase Transition, Particle Creation, Relativity

\section{Introduction}

Though it is generally accepted that the Newtonian constant of gravity $G$ plays the role of a coupling constant between geometry and matter in the Einstein field equations, it appears natural to look at this constant as a function of time in an evolving universe. There are extensions of Einstein's theory of gravitation in which $G$ is taken to vary with time [1]. In general relativity $G$ plays the role of a coupling constant between geometry and matter in Einstein's field equations. The value of $G$ is to be constant, since $G$-constancy is in-built as a manifestation of the principle of equivalence. A breakdown from the principle of equivalence, in any form, would constitute a departure from Einstein's general relativity. The time-dependence of $G$ follows as a natural consequence of Dirac's large number hypothesis [2]. The implication of time-varying $G$ will become more important at the early stage of the evolution of the universe.

[3] studied the homogeneous and isotropic cosmological model in which the parameter gamma of "Gammalaw" equation of state $p=(\gamma-1) \rho$ varies continuously with cosmic time $t$. He studied the evolution of the universe as it goes from an inflationary phase to a radiationdominated phase. [4] obtained a singularity-free model of the evolving universe with matter and studied the transition from the beginning to the radiation-dominated and matter-dominated periods of the universe.

Some workers studied the problem of the universe by linking the variation of $G$ with that of the cosmological constant $\Lambda$ leaving the form of the field equations unchanged and preserving the conservation of the energymomentum tensor of the matter content by [5-8]. The possibility of an increasing $G$ was also suggested by [9]. The possibility of the creation field with $G$ varying as some powers of $t$ discussed by [8]. [10] also presented exact solutions for zero pressure Robertson-Walker cosmological models with $G$ varying as some powers of R. A spatially flat FRW model wherein the vacuum energy density has been taken to vary as the radiation energy density discussed by $[11,12]$ discussed about the effect of bulk viscosity on the early evolution of universe, [13] discussed about early universe with variable cosmological and gravitational constants in higher dimensional space time. $[14,15]$ discussed about the cosmology of the very early universe and structures in the Universe by exact methods.

Here in this problem we study the evolution of the universe taking the Robertson-Walker models of radiating 
perfect fluid distribution in the presence of zero-mass scalar field in which the gravitational parameter $G$ varies with cosmic time t. Solutions are obtained for inflationary phase and radiation-dominated phase. The physical behaviors of the cosmological solutions are discussed. Also we study the effects of incorporating a zero-mass scalar field which is operative during an instantaneous phase transition, and it is found that the presence of the scalar field is instrumental in avoiding the initial singularity and gives freedom to the choice of initial conditions, thus giving advantage of studying different scenarios. Its presence also results in particle creation and thereby influence the subsequent evolution of the universe find Robertson-Walker models starting either from a non-singular origin with a minimum, non-zero $G$ or from a singularity with a vanishing $G$, where $G$ increases continuously in the radiation dominated era and approaches a constant value as the universe turns matter-dominated, finally the models approaching the standard model. Such study of the evolution of the universe will be of great importance in revealing the many mysteries of the different astrophysical objects in general and the universe in particular.

\section{Field Equations}

The line element considered for this problem is

$$
\mathrm{d} s^{2}=\mathrm{d} t^{2}-R^{2}(t)\left[\frac{\mathrm{d} r^{2}}{1-k r^{2}}-r^{2} \mathrm{~d} \theta^{2}+r^{2} \sin ^{2} \theta \mathrm{d} \varphi^{2}\right]
$$

where $R(t)$ is the scale factor and $K$, the curvature index which takes values $+1,0$ and -1 .

Considering perfect fluid distribution coupled with zero-rest mass scalar field the energy-momentum tensor takes the form

$$
T_{i j}=P_{i j}+S_{i j},
$$

where $P_{i j}$ is the energy-momentum tensor due to a perfect fluid given by

$$
P_{i j}=(\rho+p) u_{i} u_{j}-p g_{i j}, \quad u_{i} u^{i}=1
$$

And $S_{i j}$ is the energy-momentum tensor due to zeromass scalar field given by

$$
S_{i j}=\frac{1}{4 \pi}\left[\phi_{i} \phi_{j}-\frac{1}{2} g_{i j} \phi_{\alpha} \phi^{\alpha}\right]
$$

Where the scalar potential $\phi$ satisfies the equation

$$
g^{i j} \phi_{i j}=0
$$

Thus from the Einstein field equation

$$
R_{i j}-\frac{1}{2} g_{i j} R=-8 \pi G(t) T_{i j}
$$

We get

$$
2 \frac{\ddot{R}}{R}+\frac{\dot{R}^{2}}{R^{2}}+\frac{k}{R^{2}}=-8 \pi G p-G \phi^{2}
$$

and

$$
\frac{3 \dot{R}^{2}}{R^{2}}+\frac{3 k}{R^{2}}=8 \pi G \rho+G \dot{\phi}^{2} .
$$

Now Equations (5) and (6) give

$$
\frac{\ddot{R}}{R}=-\frac{4}{3} \pi G(\rho+3 p)-\frac{2}{3} G \dot{\phi}^{2}
$$

and

$$
R \ddot{R}+2\left(\dot{R}^{2}+k\right)=4 \pi G(\rho-p) R^{2}
$$

From Equation (4) we have

$$
\ddot{\phi}+\frac{3 \dot{R}}{R} \dot{\phi}=0,
$$

Which give us

$$
\dot{\phi}=\ell R^{-3}
$$

where $\ell$ is an integration constant.

Again from Equations (7) and (8) we have, eliminating $\ddot{R}$,

$$
\frac{\dot{R}^{2}}{R^{2}}+\frac{k}{R^{2}}=\frac{8}{3} \pi G \rho+\frac{1}{3} G \dot{\varphi}^{2}
$$

For the radiating universe we have the relation

$$
p=\frac{\rho}{3}
$$

Thus Equations (8)-(10) give

$$
\frac{\ddot{R}}{R}+2 \frac{\dot{R}^{2}}{R^{2}}+2 \frac{k}{R^{2}}=8 \pi G p
$$

and

$$
\frac{\dot{R}^{2}}{R^{2}}+\frac{k}{R^{2}}-\frac{\ell^{2} G}{3 R^{6}}=8 \pi G p .
$$

Now from Equations (12) and (13) we have

$$
R \ddot{R}+\dot{R}^{2}=-\left(k+\frac{1}{3} \ell^{2} G R^{-4}\right)
$$

We consider underneath the different phases and scenario in the course of evolution of the universe.

\section{Case I}

$$
R^{2}(t)=\left\{\begin{array}{lr}
M_{l} t-\left[k+\frac{\ell^{2} N}{3}\right] t^{2}+M_{2}, & 0 \leq t<t_{c} \\
M_{3}\left(t-t_{c}\right)-\left[k+\frac{\ell^{2} N}{3}\right]\left(t-t_{c}\right)^{2}+M_{4}, & t_{c} \leq t \leq t_{f}
\end{array}\right.
$$

with

$$
G=N R^{4} .
$$

Thus we have here 


$$
H=\frac{\dot{R}}{R}= \begin{cases}\frac{\frac{M_{l}}{2}-\left[k+\frac{\ell^{2} N}{3}\right] t}{M_{l} t-\left[k+\frac{\ell^{2} N}{3}\right] t^{2}+M_{2}} & 0 \leq t<t_{c} \\ \frac{M_{3}}{2}-\left[k+\frac{\ell^{2} N}{3}\right]\left(t-t_{c}\right) & t_{c} \leq t \leq t_{f} \\ M_{3}\left(t-t_{c}\right)-\left[k+\frac{\ell^{2} N}{3}\right]\left(t-t_{c}\right)^{2}+M_{4} & \end{cases}
$$

where in this paper $t_{c}$ means the time at which the critical temperature occurs, that is at which a phase transition occurs and $t_{f}$ the time at which that particular phase ends.

\section{Case I (a): An Inflationary Phase}

Now taking the initial condition as $R=R_{o}$ at $t=0$, we obtain from Equation (15)

$$
R_{o}^{2}=M_{2}
$$

And from relation (18) we get

$$
H_{o}=\frac{M_{l}}{2 R_{o}^{2}}
$$

but

$$
H_{0}=\frac{\dot{R}_{0}}{R_{0}}=0 \quad \text { as } \quad \dot{R}_{0}=0,
$$

since $R_{0}$ is a particular value of $R$ at $t=0$.

Thus Equation (10) with $H_{o}=0$ reduces to

$$
\left(3 k-B \ell^{2}\right) R_{o}^{-2}=8 \pi G \rho_{o} \geq 0
$$

which shows that $k \geq \frac{B \ell^{2}}{3}>0$ and thus $k=1$.

Therefore we get

$$
3 \geq B \ell^{2}>0
$$

Equation (16) with the condition $R(t)=R_{c}$, at $t=t_{c}$ gives $M_{4}=R_{c}^{2}$. And the continuity for $R(t)$ across $t=t_{c}$ implies

$$
R_{o}^{2}=R_{c}^{2}+\left[\frac{\ell^{2} N}{3}+1\right] t_{c}^{2} .
$$

Now relations (18) and (19) give, in the limit $t \rightarrow t_{c}$

$$
H^{-}\left(t_{c}\right)=-\frac{\left[\frac{\ell^{2} N}{3}+1\right] t_{c}}{R_{c}^{2}}
$$

and

$$
H^{+}\left(t_{c}\right)=\frac{M_{3}}{2 R_{c}^{2}}
$$

Thus the continuity in $H$ due to phase transition, which arises due to presence of scalar field, is found to be

$$
\Delta H=H^{+}\left(t_{c}\right)-H^{-}\left(t_{c}\right)=\frac{A_{3}+2\left[\frac{\ell^{2} N}{3}+1\right] t_{c}}{2 R_{c}^{2}}
$$

Since $\Delta H$ must vanish in the limit, we have

$$
M_{3}=-2\left[\frac{\ell^{2} N}{3}+1\right] t_{c} .
$$

Hence Equations (15) and (16) reduce to (see (27)-(28)).

Equations (27)-(28) show that the expansion is endowed with a generalized inflation $(\ddot{R}>0)$ as long as $\dot{R}$ remains greater than

$$
\left[\frac{\ell^{2} N}{3}+1\right]^{1 / 2}
$$

and turns deflationary as $\dot{R}$ becomes smaller than

$$
\left[\frac{\ell^{2} N}{3}+1\right]^{1 / 2} \text {. }
$$

\section{Case I (b) A Radiation-Dominated Phase}

Now Equation (4) gives

$$
R(t)= \begin{cases}{\left[R_{o}^{2}-\left[\frac{\ell^{2} N}{3}+1\right] t^{2}\right]^{1 / 2}=\left[R_{c}^{2}+\left[\frac{\ell^{2} N}{3}+1\right]\left(t_{c}^{2}-t^{2}\right)\right]^{1 / 2},} & 0 \leq t<t_{c} \\ {\left[R_{c}^{2}-\left[\frac{\ell^{2} N}{3}+1\right]\left(t-t_{c}\right)\left(t+t_{c}\right)\right]^{1 / 2},} & t_{c} \leq t \leq \infty\end{cases}
$$




$$
\ddot{\varphi}+3 \frac{\dot{R}}{R} \dot{\varphi}=0 .
$$

Also Equations (7) and (10) respectively give

$$
\dot{H}+H^{2}=-\frac{4}{3} \pi \dot{G}(\rho+3 p)-\frac{2}{3} G \dot{\phi}^{2}
$$

and

$$
H^{2}+\frac{k}{R^{2}}=\frac{8}{3} \pi G \rho+\frac{1}{3} G \dot{\phi}^{2}
$$

where $H=\frac{\dot{R}}{R}$ is the Hubble's parameter.

Thus from Equations (11) and (30) we get

$$
\dot{H}+H^{2}=-\frac{8}{3} \pi G \rho-\frac{2}{3} G \dot{\varphi}^{2}
$$

Now eliminating $\rho$ between Equations (31) and (32) we have

$$
\dot{H}+2 H^{2}+\frac{k}{R^{2}}+\frac{1}{3} G \dot{\varphi}^{2}=0
$$

which can be rewritten as

$$
\begin{gathered}
\frac{\dot{H}}{R}+\frac{2 H^{2}}{R}+\frac{k}{R^{3}}+\frac{1}{3} \frac{G}{R} \dot{\phi}^{2}=0 \\
\text { i.e. } \frac{1}{R} \frac{\partial H}{\partial t}+\frac{2 H^{2}}{R}+\frac{k}{R^{3}}+\frac{1}{3} \frac{G}{R} \dot{\phi}^{2}=0 \\
\text { i.e. } \frac{1}{R} \frac{\partial H}{\partial R} \frac{\partial R}{\partial t}+\frac{2 H^{2}}{R}+\frac{k}{R^{3}}+\frac{1}{3} \frac{G}{R} \dot{\phi}^{2}=0 \\
\text { i.e. } \frac{1}{R} H^{\prime} \dot{R}+\frac{2 H^{2}}{R}+\frac{k}{R^{3}}+\frac{1}{3} \frac{G}{R} \dot{\phi}^{2}=0 \\
\text { i.e. } \frac{\dot{R}}{R} H^{\prime}+\frac{2 H^{2}}{R}+\frac{k}{R^{3}}+\frac{1}{3} \frac{G}{R} \dot{\phi}^{2}=0 \\
\text { i.e. } H H^{\prime}+\frac{2 H^{2}}{R}+\frac{k}{R^{3}}+\frac{G \dot{\varphi}^{2}}{3 R}=0,
\end{gathered}
$$

where a dash denotes differentiation w.r.t. R.

Considering zero-curvature Robertson-Walker metric, Equation (34) takes the form

$$
H^{\prime}+2 \frac{H}{R}+\frac{G \dot{\varphi}^{2}}{3 H R}=0 .
$$

Now from relation (29) we get

$$
\dot{\phi}=\ell R^{-3} \text {, }
$$

where $\ell$ is integration constant.

Making use of relation (36) in Equation (35) we get

$$
H^{\prime}+2 \frac{H}{R}+\frac{\ell^{2} G}{3 H R^{7}}=0 \text {. }
$$

Without loss of generality we take the solution of
Equation (37) as

$$
G=m\left(H R^{3}\right)^{2}
$$

with

$$
H=c R^{-\left[2+\frac{\ell^{2} m}{3}\right]}
$$

where $m$ and $c$ are arbitrary constants.

If $H=H_{o}$ for $R=R_{o}$ then we get, from Equation (39)

$$
\mathrm{C}=H_{o} R_{o}^{\left(2+\frac{\ell^{2} m}{3}\right)}
$$

Thus Equation (39) takes the form

$$
H=H_{o} R_{o}^{\frac{l}{c_{o}}} R^{\frac{l}{c_{o}}}
$$

where $\left[2+\frac{\ell^{2} m}{3}\right]=\frac{l}{c_{o}}$,

Now Equation (40) gives

$$
R=\left[\frac{l}{c_{o}}\left(H_{o} R_{o}^{\frac{l}{c}} t+c_{t}\right)\right]^{c_{o}}
$$

Thus we have

$$
H=H_{o} R_{o}^{\frac{l}{c_{o}}}\left[\frac{l}{c_{o}}\left[H_{o} R_{o}^{\frac{l}{c_{o}}} t+c_{l}\right]\right]^{-1}
$$

And from relations (38), (41) and (42) we get

$$
G=m H_{o}^{2} R_{o}^{\frac{2}{c_{o}}}\left[\frac{l}{c_{o}}\left[H_{o} R_{o}^{\frac{l}{c_{o}}} t+c_{l}\right]\right]^{6 c_{o}-2}
$$

Also we have

$$
\phi=\ell c_{o}^{3 c_{o}}\left(1-3 c_{o}\right)^{-1} H_{o} R_{o}^{\frac{l}{c_{o}}}\left(H_{o} R_{o}^{\frac{l}{c_{o}}} t+c_{l}\right)^{\left(l-3 c_{o}\right)}
$$

and

$$
\begin{aligned}
& p=\frac{1}{8 \pi}\left[\frac{l}{m}-\frac{\ell^{2}}{3}\right]\left[\frac{l}{c_{o}}\left(H_{o} R_{o}^{\frac{l}{c_{o}}} t+c_{l}\right)\right]^{-6 c_{o}} \\
& \rho=\frac{3}{8 \pi}\left[\frac{l}{m}-\frac{\ell^{2}}{3}\right]\left[\frac{l}{c_{o}}\left(H_{o} R_{o}^{\frac{l}{c_{o}}} t+c_{l}\right)\right]^{-6 c_{o}}
\end{aligned}
$$

Here in this case, $G$ is found to be an increasing function of time, whereas $\phi$ is seen to be a decreasing function of time. In this phase or episode the pressure and density of the universe are found to decrease along with the increase of the cosmic age (of course, if the constants $\ell$ and $m$ are so related that $3>\ell^{2} m$ ). For this era $R$ is seen to be an increasing function of time thus showing that during this era the universe is expanding.

\section{Case II}

Now another solution of Equation (14) is 


$$
R^{2}(t)= \begin{cases}A_{l} t-\left[k-\frac{\ell^{2}}{6} N\right] t^{2}+A_{2}, & 0 \leq t<t_{c} \\ A_{3}\left(t-t_{c}\right)-\left[k \frac{\ell^{2}}{-6} N\right]\left(t-t_{c}\right)^{2}+A_{4}, & t_{c} \leq t \leq t_{f}\end{cases}
$$

with

$$
G=B\left[2\left(k-\frac{\ell^{2}}{6} N\right) t-A_{l}\right]^{-1} R^{5} \dot{R}
$$

This solution is quite different from the solution in Case-I; more particularly the value of $G$ in this case is

$$
H=\frac{\dot{R}}{R}= \begin{cases}\frac{A_{l}-2\left[k-\frac{\ell^{2}}{6} B\right] t}{2\left[A_{l} t-\left[k-\frac{\ell^{2}}{6} B\right] t^{2}+A_{2}\right]}, & 0 \leq t<t_{c} \\ \frac{A_{3}-2\left[k-\frac{\ell^{2}}{6} B\right]\left(t-t_{c}\right)}{2\left[A_{3}\left(t-t_{c}\right)-\left[k-\frac{\ell^{2}}{6} B\right]\left(t-t_{c}\right)^{2}+A_{4}\right]}, & t_{c} \leq t \leq t_{f}\end{cases}
$$

\section{Case II (a) An Inflationary Type Solution}

Taking the initial condition $R=R_{o}$ at $t=0$, we obtain from relation (48)

$$
R_{o}^{2}=A_{2} \text {. }
$$

And from relation (51) we get

$$
H_{O}=\frac{A_{l}}{2 A_{2}}=\frac{A_{l}}{2 R_{o}^{2}}
$$

Equation (10) with $H_{o}=0$ reduces to

$$
\frac{k}{R_{o}^{2}}=\frac{8}{3} \pi G \rho_{o}-\frac{1}{3} B A_{l}^{-l} \ell^{2} H_{o}
$$

Thus

$$
\frac{k}{R_{o}^{2}}=\frac{8}{3} \pi G \rho_{o}>0 .
$$

This shows that $k>0$ which implies $k=1$.

Again Equation (49) with the condition $R(t)=R_{c}$ at $t$

$=t_{c}$ give $A_{4}=R_{c}^{2}$.

And for continuity of $R(t)$ across $t=t_{c}$ we must have

$$
\begin{aligned}
R_{o}^{2}=R_{c}^{2}+\left[1-\frac{\ell^{2}}{6} B\right] t_{c}^{2} & \text { Then Equations (48) } \\
R(t) & =\left\{\begin{array}{l}
{\left[R_{o}^{2}+\left[\frac{\ell^{2}}{6} B-1\right] t^{2}\right]^{1 / 2}=\left[R_{c}^{2}-\left[\frac{\ell^{2}}{6} B-1\right]\left(t_{c}^{2}-t^{2}\right)\right]^{1 / 2}} \\
{\left[R_{c}^{2}+\left[\frac{\ell^{2}}{6} B-1\right]\left(t_{c}+t\right)\left(t-t_{c}\right)\right]^{1 / 2}}
\end{array}\right.
\end{aligned}
$$


Thus showing that the expansion is endowed with a generalized inflation $(\ddot{R}>0)$ as long as $\dot{R}$ remains smaller than

$$
\left[\frac{\ell^{2}}{6} B-1\right]^{1 / 2}
$$

and turns deflationary as $\dot{R}$ becomes larger than

$$
\left[\frac{\ell^{2}}{6} B-1\right]^{1 / 2}
$$

Also from Equation (61) we see that $R \rightarrow \infty$ as $t \rightarrow \infty$ although $k=1$. It may be taken as a significant deviation from the standard model which is resulted from the coupling of the scalar field.

Also in this case the radiation density is found to be

$$
\rho(t)=\left\{\begin{array}{l}
\frac{3}{8 \pi G}\left[\frac{3+3\left[\frac{\ell^{2}}{6} B-1\right]^{2} t^{2}\left\{R_{c}^{2}-\left[\frac{\ell^{2}}{6} B-l\right]\left(t_{c}^{2}-t^{2}\right)\right\}^{-1}-l^{2} B\left[\frac{\ell^{2}}{6} B-1\right]\left\{2\left[1-\frac{\ell^{2} B}{6}\right]\right\}}{3\left\{R_{c}^{2}-\left[\frac{\ell^{2} B}{6}-1\right]\left(t_{c}^{2}-t\right)\right\}}\right], \quad 0 \leq t<t_{c}, \\
\frac{3}{8 \pi G}\left[\frac{3+3\left[\frac{\ell^{2} B}{6}-1\right] t^{2}\left\{R_{c}^{2}+\left[\frac{\ell^{2} B}{6}-1\right]\left(t_{c}+t\right)\left(t-t_{c}\right)\right\}^{-1}-\ell^{2} B\left[\frac{\ell^{2} B}{6}-1\right]\left\{2\left[1-\frac{\ell^{2} B}{6}\right] t\right\}}{3\left\{R_{c}^{2}+\left[\frac{\ell^{2} B}{6}-1\right]\left(t_{c}+t\right)\left(t-t_{c}\right)\right\}}\right], \quad t_{c} \leq t \leq \infty,
\end{array}\right.
$$

\section{Case II (b) Big Bang Scenario}

Considering the condition $R(t)=0$ at $t=0$ and $R(t)=$ $R_{c}$ at $t=t_{c}$,Equations (48) and (49) give

$$
A_{2}=0 \text {. }
$$

and

$$
A_{4}=R_{c}^{2} .
$$

Thus for the continuity of $R(t)$ at $t=t_{c}$ we must have

$$
A_{l}=\left[R_{c}^{2}+\left[k-\frac{\ell^{2} B}{6}\right] t_{c}^{2}\right] t_{c}^{-1}
$$

Therefore for the limit $t \rightarrow t_{c}$ relations (51) and (52) become

$$
H^{-}\left(t_{c}\right)=\frac{R_{c}^{2} t_{c}^{-1}-\left[k-\frac{\ell^{2} B}{6}\right] t_{c}}{2 R_{c}^{2}}
$$

and

$$
H^{+}\left(t_{c}\right)=\frac{A_{3}}{2 R_{c}^{2}}
$$

Thus the continuity in $H(t)$ due to the phase transition follows from

$$
\Delta H=H^{+}\left(t_{c}\right)-H^{-}\left(t_{c}\right)=\frac{A_{3} t_{c}-R_{c}^{2}+\left[-\frac{\ell^{2}}{6} B\right] t_{c}^{2}}{2 R_{c}^{2} t_{c}}
$$

Therefore for $\Delta H \rightarrow 0$ we obtain

$$
A_{3}=\left[R_{c}^{2}-\left[k-\frac{\ell^{2}}{6} B\right] t_{c}^{2}\right] t_{c}^{-2}
$$

Hence we have

$$
R(t)= \begin{cases}{\left[R_{c}^{2}+\left[k-\frac{\ell^{2}}{6} B\right] t_{c}\left(t_{c}-t\right)\right]^{\frac{1}{2}}\left[\frac{t}{t_{c}}\right]^{\frac{1}{2}},} & 0 \leq t<t_{c} \\ {\left[\left\{R_{c}^{2}-\left[k-\frac{\ell^{2}}{6} B\right] t_{c}^{2}\right\}\left(t-t_{c}\right) t_{c}^{-1}-\left[k-\frac{\ell^{2}}{6} B\right]\left(t-t_{c}\right)^{2}+R_{c}^{2}\right]^{\frac{1}{2}},} & t_{c} \leq t \leq t_{f}\end{cases}
$$

Underneath we shall study two different types of evolution one having the phase transition in the expanding phase and other in the collapsing phase depending upon whether $R_{c}>t_{c}$ or $R_{c}<t_{c}$.

\section{Case II (b)*}

We see from Equations (72) and (73) that the universe reaches its maximum radius at

$$
t_{\max }=t_{c}+\frac{\left\{R_{c}^{2}-\left[l-\frac{\ell^{2}}{6} B\right] t_{c}^{2}\right\}}{2\left[l-\frac{\ell^{2}}{6} B\right] t_{c}}>t_{c}
$$




$$
R_{\max }=\left[R_{c}^{2}+\frac{\left\{R_{c}^{2}-\left[l-\frac{\ell^{2}}{6} B\right] t_{c}^{2}\right\}^{2}}{4\left[l-\frac{\ell^{2}}{6} B\right] t_{c}^{2}}\right]^{\frac{1}{2}}
$$

Then after that it collapses to a singularity at $t=t_{f}$ give by

$$
t_{f}=t_{\max }+\frac{\left[\left\{R_{c}^{2}-\left[l-\frac{\ell^{2}}{6} B\right] t_{c}^{2}\right\}^{2}+4\left[l-\frac{\ell^{2}}{6} B\right] R_{c}^{2}\right]^{\frac{1}{2}}}{2\left[l-\frac{\ell^{2}}{6} B\right] t_{c}}
$$

Here we see that the phase transition occurs in the expanding phase of evolution (before reaching the maximum radius) at $t=t_{c}$ when there is a sudden rise in the rate of expansion. And the subsequent evolution is affected by the presence of the scalar field. The larger the value of $\ell$ or in other words greater the strength of the scalar field the longer it will take for the universe to reach its maximum radius, and in this way the final collapse will be prolonged.

Case II (b)**

Here we take up the case

$$
0<B<\frac{6}{\ell^{2}} \text { with } R_{c}<\left[l-\frac{\ell^{2}}{6} B\right]^{\frac{1}{2}} t_{c} .
$$

In this case we see that the radius reaches a maximum before the transition takes place, as is clear from the examination of the Equations (72) and (73). This gives

$$
t_{\max }=\frac{t_{c}}{2}+\frac{R_{c}^{2}}{2\left[l-\frac{\ell^{2}}{6} B\right] t_{c}}<t_{c}
$$

and

$$
R_{\max }=\left[\frac{R_{c}^{4}+3 t_{c}^{4}\left[l-\frac{\ell^{2}}{6} B\right]^{2}-2 R_{c}^{2} t_{c}^{2}\left[l-\frac{\ell^{2}}{6} B\right]}{4\left[l-\frac{\ell^{2}}{6} B\right] t_{c}^{2}}\right]^{\frac{1}{2}}
$$

Here the universe seems to collapse to a singularity at

$$
t_{f}=t_{c}+\frac{\left[\left\{R_{c}^{2}-\left[l-\frac{\ell^{2} B}{6}\right] t_{c}^{2}\right\}^{2}+4\left[l-\frac{\ell^{2} B}{6}\right] R_{c}^{2} t_{c}^{2}\right]^{\frac{1}{2}}-\left\{\left[l-\frac{\ell^{2} B}{6}\right] t_{c}^{2}-R_{c}^{2}\right\}}{2\left[l-\frac{\ell^{2} B}{6}\right] t_{c}}
$$

It is observed that the transition takes place in the collapsing phase of evolution $(\theta<0)$ and the presence of scalar field therefore adds to the hydrostatic pressure (as $p^{\prime}>0$ in the collapsing phase), and thereby to the inertial effects which make the universe to collapse back to singularity at a faster rate as clear from Equation (70).

Moreover, the time variation of energy density in the different models may be obtained from Equation (10) by using the time dependence of the functions $R(t)$ and $H(t)$.

Thus we have here

$$
\begin{aligned}
& \rho(t)=\frac{1}{32 \pi G\left\{R_{c}^{2}+\left(6-\ell^{2} B\right) t_{c}\left(t_{c}-t\right)\right\}^{2}} \times\left[3\left\{R_{c}^{2}+\left(6-\ell^{2} B\right) t_{c}\left(t_{c}-t\right)\right\}\left\langle\left\{R_{c}^{2}+\left(6-\ell^{2} B\right) t_{c}\left(t_{c}-t\right)\right\} t^{-2}+t_{c} t^{-1}\right\rangle\right. \\
& -3 t_{c}^{2}\left(6-\ell^{2} B\right)^{2}-\ell^{2} B\left\{R_{c}^{2}+\left(6-\ell^{2} B\right) t_{c}\left(t_{c}-t\right)\right\} \times\left\{\begin{array}{l}
\left(6-\ell^{2} B\right)^{-1} t_{c}^{-1} t^{-2}\left\{R_{c}^{2}+\left(6-\ell^{2} B\right) t_{c}\left(t_{c}-t\right)\right\}-t_{c} t^{-1}- \\
2\left\{R_{c}^{2}+\left(6-\ell^{2} B\right) t_{c}^{2}\right\}^{-1}\left\{1-t_{c}^{2}\left(6-\ell^{2} B\right)\right\}
\end{array}\right]
\end{aligned}
$$

where

$$
\begin{aligned}
\rho(t)= & \frac{1}{32 \pi G\left\{R_{c}^{2}\left(t-t_{c}\right) t_{c}^{-1}-\left(6-\ell^{2} B\right)\left(t-t_{c}\right) t_{c}-\left(6-\ell^{2} B\right)\left(t-t_{c}\right)^{2}+R_{c}^{2}\right\}^{2}} \\
& \times\left[3\left\{R_{c}^{2} t_{c}^{-1}+\left(6-\ell^{2} B\right) t_{c}+\left(6-\ell^{2} B\right) t_{c}-2\left(6-\ell^{2} B\right) t\right\}+\left\{R_{c}^{2}\left(t-t_{c}\right) t_{c}^{-1}-\left(6-\ell^{2} B\right)\left(t-t_{c}\right) t_{c}-\left(6-\ell^{2} B\right)\left(t-t_{c}\right)^{2}+R_{c}^{2}\right\}\right. \\
& \times\left(\begin{array}{l}
12-\ell^{2} B\left\{R_{c}^{2} t_{c}^{-1}+\left(6-\ell^{2} B\right) t_{c}-2\left(6-\ell^{2} B\right) t\right\} \\
\left\{\left(6-\ell^{2} B\right) t\right\}^{-1}+2 \ell^{2} B t_{c}\left\{R_{c}^{2} t_{c}^{-1}+\left(6-\ell^{2} B\right) t_{c}-2\left(6-\ell^{2} B\right) t\right\}\left\{R_{c}^{2}+\left(6-\ell^{2} B\right) t_{c}^{2}\right\}^{-1}
\end{array}\right]
\end{aligned}
$$


where

$$
\begin{array}{r}
t_{c} \leq t \leq t_{f} \quad(81) \quad \text { phase transition occurring at } t=t_{c} \text { is given by } \\
\Delta \rho=\frac{R_{c}^{-4}}{32 \pi G}\left[\begin{array}{l}
3 R_{c}^{2} t_{c}^{-1}\left(t_{c}^{-1}-l\right)+\ell B\left\langle t_{c}^{-2}\left(6-\ell^{2} B\right)^{-1}\left\{2 R_{c}^{2}-R_{c}^{4}\left(t_{c}^{-1}+l\right)+2 t_{c}^{4}\right\}-2 t_{c}\left(6-\ell^{2} B\right)-2 R_{c}^{2}\right\rangle \\
-9 R_{c}^{2}+3\left(6-\ell^{2} B\right) t_{c}\left\{1-\left(6-\ell^{2} B\right) t_{c}\right\}
\end{array}\right]
\end{array}
$$

Therefore, the discontinuity in the energy due to the

\section{Conclusions}

The models we obtain here are interesting as they ultimately evolve to the standard big bang models in the present era though their early scenarios are altogether different. Here solutions are obtained for spatially homogeneous and isotropic perfect fluid cosmological model in the presence of zero-mass scalar field which is one of the most acceptable models of the present universe. A unified description of early evolution of the universe is studied for two different periods where the gravitational constant is allowed to depend on cosmic time t. The models are expanding ones in each phase of evolution, and the solutions obtained in each phase are identically satisfied.

In case II (b)* we see that the phase transition occurs in the expanding phase of evolution (before reaching the maximum radius) at $t=t_{c}$ when there is a sudden rise in the rate of expansion. And the subsequent evolution is affected by the scalar field. The larger the value of $\ell$ or in other words greater the strength of the scalar field the longer it will take for the universe to reach its maximum radius; and in this way the final collapse will be prolonged.

In Case II (b)** the model for

$$
B=\frac{6}{\ell^{2}}
$$

with $k=1$ is an interesting one where the evolution for $t<t_{c}$ is akin to that of a standard Big Bang model with flat special sections. At $t=t_{c}$, the Hubble parameter suffers a sudden rise in its value which is due to a decrease in the hydrostatic pressure arising out of the presence of the scalar field. The subsequent evolution, therefore, starts with an increased rate of expansion. Here it is the presence of the scalar field which is instrumental in making the geometrically closed models ever expanding. Moreover relation (82) indicates that there is an instantaneous rise in the energy density after the phase transition in all $(k=1,-1,0)$ big bang models, which may be interpreted as on account of generation of particles due to presence of scalar field.

In case II (b) where a radiation-dominated phase is considered we see that both $\mathrm{R}$ and $\mathrm{H}$ are increasing functions of time thus showing that our universe is an expanding one. And also $G$ is seen to be continuously in- creasing in this radiation-dominated phase.

Here the scalar field is found to exist only for $1>3 c_{0}$. Moreover the condition $1>3 c_{0}$ implies that the deceleration parameter $\mathrm{q}$ is greater than 2 . Also in this case the pressure and energy density are found to be decreasing functions of time which shows that the evolving universe comes out to be one of the realistic models.

\section{References}

[1] P. S. Wesson, "Gravity, Particles \& Astrophysics," D. Reidel, Dordrecht, 1980.

[2] P. A. M. Dirac, “A New Basis for Cosmology,” Proceedings of the Royal Society of London, Series A, Mathematical and Physical Sciences, Vol. 165, No. 921, pp. 199208.

[3] J. C. Carvalho, "Unified Description of the Early Universe," International Journal of Theoretical Physics, Vol. 35, No. 9, 1996, pp. 2019-2028. doi:10.1007/BF02302426

[4] M. Israclit and N. Rosen, "A Singularity-Free Cosmological Model in General Relativity," Astrophysical Journal, Vol. 342, 1989, pp. 627-632. doi:10.1086/167622

[5] D. Kalligas, P. Wesson and C. W. F. Everitt, "Flat FRW Models with Variable G and $\Lambda$," General Relativity and Gravitation, Vol. 24, No. 4, 1992, pp. 351-357. doi:10.1007/BF00760411

[6] A. M. M. Abdel Rahman, "A Critical Density Cosmological Model with Varying Gravitational and Cosmological 'Constants'," General Relativity and Gravitation, Vol. 22, No. 6, 1990, pp. 655-657. doi:10.1007/BF00755985

[7] M. S. Berman, "Cosmological Models with a Variable Cosmological Term," Physical Review D, Vol. 43, No. 4, 1991, pp. 1075-1078. doi:10.1103/PhysRevD.43.1075

[8] A. Beesham, "Variable-G Cosmology and Creation," International Journal of Theoretical Physics, Vol. 25, No. 12, 1986, pp. 1295-1298. doi:10.1007/BF00670415

[9] L. S. Levit, "The Gravitational Constant at Time Zero," Lettere Al Nuovo Cimento, Vol. 29, No. 1, 1980, pp. 2324. doi:10.1007/BF02745337

[10] R. F. Sistero, "Cosmology with G and $\Lambda$ Coupling Scalars," General Relativity and Gravitation, Vol. 23, No. 11, 1991, pp. 1265-1278. doi:10.1007/BF00756848

[11] K. Freese, F. C. Adams, J. A. Frieman and E. Mottola, "Cosmology with Decaying Vaccum Energy," Nuclear Physics B, Vol. 287, 1987, pp. 797-814. doi:10.1016/0550-3213(87)90129-5 
[12] C. P. Singh, "Bulk Viscous Cosmology in Early Universe," Pramana, Vol. 71, No. 1, 2008, pp. 33-48. doi:10.1007/s12043-008-0139-4

[13] G. S. Khadekar, et al. "Early Viscous Universe with Variable Cosmological and Gravitational Constants in Higher Dimensional Space Time," Physics and Astronomy, Vol. 47, No. 11, 2008, pp. 3057-3074. doi:10.1007/s10773-008-9741-y
[14] R. H. Brandenberger, "Cosmology of the Very Early Universe, 8 March 2010. arxiv: 1003. 1745VI [hep-th].

[15] C. G. Bohmer, K. Bolejko, A. Krasi'nski, C. Hellaby, M.-N. Célérier, "Structures in the Universe by Exact Methods," Cambridge University Press, 2009, p. 256, GBP80.00, USD124.00, ISBN-13: 9780521769143 General Relativity and Gravitation, Vol. 43, No. 3, 2011, pp. 923-924. doi:10.1007/s10714-010-1126-x 